\title{
Earlier decline in sniff nasal inspiratory pressure than peak expiratory flow in children with Duchenne muscular dystrophy
}

\begin{abstract}
To the Editor:
In Duchenne muscular dystrophy (DMD), progressive weakness of the respiratory muscles leads to a restrictive ventilatory defect contributing to early morbidity and mortality. We have recently shown by a longitudinal assessment of vital capacity and sniff nasal inspiratory pressure (SNIP) in young DMD children that SNIP was an earlier marker of decline in respiratory muscle strength than vital capacity [1]. However, early involvement of the expiratory muscles is a characteristic feature of DMD, as shown by the earlier decrease in expiratory (P Emax $_{\text {man }}$ than inspiratory maximal pressure (PImax) [2]. In the absence of bronchial obstruction, peak expiratory flow (PEF) reflects maximal expiratory muscle strength [3]. In patients with respiratory weakness, PEF is reduced and correlated with PEmax [4]. Recently, it was shown that DMD patients on a 1-year idebenone treatment improved in expiratory muscle strength (evaluated by PEF) while patients on placebo deteriorated [5]. No difference between treatments groups were observed for measures such as vital capacity [6], suggesting that PEF could be used as an outcome parameter to assess the effect of early therapeutic interventions on respiratory muscle strength in DMD. As candidate drugs for the treatment of DMD enter clinical trials, it is important to determine the natural evolution of pulmonary function parameters that could be used as outcome measures for efficacy studies in DMD children. However, to our knowledge, there are no data on the natural evolution of PEF in young DMD children and there is no information on the age of PEF decline compared with the age of SNIP decline. Therefore, we aimed to assess the PEF and SNIP change with age in glucocorticoid-naive DMD children in order: 1) to document the natural evolution of PEF measurement; and 2) to identify the ages of decline in PEF and SNIP in DMD children.
\end{abstract}

A 3-year, prospective follow-up of SNIP and PEF at 6-month intervals was performed in $33 \mathrm{DMD}$, steroidnaïve 5-20-year-olds [1]. Vital capacity and PImax were also assessed [1].

Inclusion criteria and SNIP assessments have been described previously [1]. At least 10 (most often 15-20) maximal sniffs, performed from functional residual capacity, were obtained. PEF was obtained from flowvolume curve data (Masterscreen Pneumo; Erich Jaeger, Wurzburg, Germany) as the maximum expiratory flow achieved from a maximum forced expiration, starting without hesitation from the point of maximal lung inflation. The largest value from at least three acceptable manoeuvres was recorded [7]. Results were expressed as z-scores $[8,9]$. Median (interquartile range (IQR)) is presented for continuous variables and percentages for categorical variables. At inclusion, for SNIP, the within-session repeatability was evaluated by the coefficient of variation $(\mathrm{CoV})$, and for PEF, by the difference between the largest and the next largest manoeuvre [7]. A nonlinear mixed model was used to analyse the change of PEF and SNIP versus a broken function of age that took 0 before the knot and subject's age after the knot [10]; the knot position was optimised by the smallest Akaike information criterion. At this optimal knot, intercept (no change) and slope (decrease) was estimated; comparison between the age of the knot for SNIP and that for PEF was performed on the z-score value of these two parameters.

The full DMD characteristics were described in our previous study; ambulation was lost at a median age of 9.4 years (IQR 8.3-10.4 years). Scoliosis developed in $61 \%$ of DMD during the follow-up; spine fusion was performed in $36 \%$ of the non-ambulatory patients with spinal curvature $>30^{\circ}$ at a median age of 13.2 years (IQR 12.5-13.8 years). Nocturnal non-invasive ventilation (NIV) had been initiated in one subject before the beginning of the study.

At inclusion, median age was 11.0 years (range 5.0-16.7 years), median height was $136 \mathrm{~cm}$ (range 103$170 \mathrm{~cm}$ ) and median body mass index (BMI) $\mathrm{z}$-score was -0.10 (range $-4.80-6.30$ ) [11]. Baseline median SNIP z-score was -2.20 (range $-4.24--0.91 ; 79 \%$ of children had values $<-1.64$ ) while baseline median PEF $\mathrm{z}$-score was -1.20 (range $-3.94-0.58 ; 34 \%$ of children had values $<-1.64$ ). SNIP baseline median CoV was 

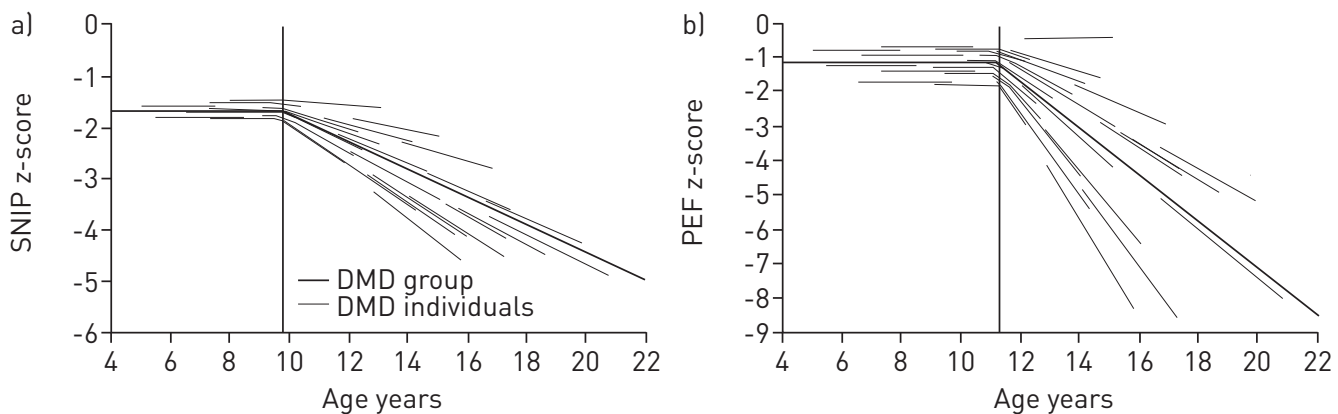

FIGURE 1 Analysis of longitudinal data. The model that best fitted the data predicted that sniff nasal inspiratory pressure (SNIP) values plateaued at a $z$-score of -1.6956 until the age of 9.72 years then declined by 0.2652 per year of age, while peak expiratory flow (PEF) z-scores plateaued at -1.1686 until 11.45 years then declined by 0.6828 per year. The SNIP values therefore declined before PEF values.

8\% (IQR 6-11\%). Median difference between the largest two PEFs was $0.050 \mathrm{~L} \cdot \mathrm{s}^{-1}\left(\mathrm{IQR} 0.040-0.190 \mathrm{~L} \cdot \mathrm{s}^{-1}\right.$ ) or $2 \%$ of highest PEF (IQR $1-6 \%)$.

The model (broken function of age) that best fitted SNIP and PEF values in longitudinal analysis is shown in figure 1, demonstrating an earlier decline in SNIP than PEF values (from 9.72 years of age ( $95 \%$ CI 8.8910.55 years) and 11.45 years of age (95\% CI 11.09-11.81); $\mathrm{p}<0.0001)$.

Although early involvement of the expiratory muscles is a characteristic feature of DMD (as shown by the earlier decrease in PEmax than PImax [2] or values of gastric pressure during cough being below normal values in patients as early as 8 years of age [12]), this longitudinal assessment of SNIP and PEF shows an earlier decline in SNIP than PEF in DMD children.

PEF baseline values in the present study showed a good repeatability (as the largest two blows were reproducible to within $0.670{\mathrm{~L} . s^{-1}}^{-1}[7]$ and as median difference between the two best measurements was $2 \%$ of the best) and appeared reliable. Our transverse data at inclusion showed that a significant decrease in inspiratory muscle pressure (SNIP z-score $<-1.64$ ) was already present in a majority $(79 \%)$ of children (median age 11 years) while only a minority them (34\%) of experienced a significant PEF decrease. As a result, median SNIP was already below normal values (z-score -2.20) while median PEF remained within normal values.

Our longitudinal data analysis adds that the decline in SNIP values began 1.73 years earlier than that of PEF.

As PEF depends not only on expiratory strength but also on inspiratory effort [13], we suggest that PEF may be relatively preserved as long as the inspiratory muscle function, the speed of inspiration (as evaluated by SNIP), the vital and inspiratory capacities, and the thoracopulmonary elastic recoil are only moderately affected [14]. We also suggest that with deterioration of both inspiratory muscle strength and vital capacity, after 11 years of age (the same broken-line model applied to the longitudinal assessment of vital capacity showed that vital capacity values were below the lower limit of normal after 11 years of age), DMD subjects may no longer be able to perform fast inspirations reaching $85-95 \%$ of their inspiratory capacity, producing increases in the elastic recoil of the lung and chest wall and, therefore, greater PEF values, and enhancing expiratory muscle pressure during the subsequent expiration [15].

SNIP is useful in the detection of inspiratory strength decline in young DMD cases when vital capacity remains within normal values. It has the advantage of being obtainable from DMD subjects as young as 5 years and could be suitable as a sensitive secondary outcome measure specifically evaluating respiratory muscle strength in young children up to 10 years of age. SNIP may also provide information regarding the severity of the clinical DMD subphenotype, as children requiring earlier NIV exhibit an earlier decrease in SNIP [1].

In conclusion, although PEF was shown to be a sensitive parameter to monitor the effect of therapeutic intervention on respiratory muscle strength in 8-16-year-old DMD children [6], our data show that SNIP decline occurred before PEF decline in young DMD children and that SNIP decline is therefore an earlier sign of the decline of respiratory muscle strength in DMD than the decline of PEF. 
Véronique Nève, Jean-Louis Edmé and Régis Matran

Service d'Explorations Fonctionnelles Respiratoires, CHRU de Lille, and Univ. Lille Nord de France, UDSL, Lille, France.

Correspondence: Véronique Nève, Service d'Explorations Fonctionnelles Respiratoires, Hôpital Calmette, CHRU de Lille

2, Avenue Oscar Lambret, 59000 Lille, France. E-mail: veronique.neve@chru-lille.fr

Received: March 062014 | Accepted after revision: June 302014 | First published online: Aug 192014

Support statement: The research was supported by a grant from the French Ministry of Health (PHRC number 2001 1937).

Conflict of interest: None declared.

\section{References}

Nève V, Cuisset JM, Edmé JL, et al. SNIP interest in the longitudinal assessment of young Duchenne muscular dystrophy children. Eur Respir J 2013; 42: 671-680.

2 Hahn A, Bach JR, Delaubier A, et al. Clinical implications of maximal respiratory pressure determinations for individuals with Duchenne muscular dystrophy. Arch Phys Med Rehabil 1997; 78: 1-6.

3 Suarez AA, Pessolano FA, Monteiro SG, et al. Peak flow and peak cough flow in the evaluation of expiratory muscle weakness and bulbar impairment in patients with neuromuscular disease. Am J Phys Med Rehabil 2002; 81: $506-511$.

4 Vincken WG, Elleker MG, Cosio MG. Flow-volume loop changes reflecting respiratory muscle weakness in chronic neuromuscular disorders. Am J Med 1987; 83: 673-680.

5 Buyse GM, Goemans N, van den Hauwe M, et al. Effects of glucocorticoids and idebenone on respiratory function in patients with duchenne muscular dystrophy. Pediatr Pulmonol 2013; 48: 912-920.

6 Buyse GM, Goemans N, van den Hauwe M, et al. Idebenone as a novel, therapeutic approach for Duchenne muscular dystrophy: results from a 12 month, double-blind, randomized placebo-controlled trial. Neuromuscul Disord 2011; 21: 396-405.

7 Miller MR, Hankinson J, Brusasco V, et al. Standardisation of spirometry. Eur Respir J 2005; 26: 319-338.

8 Stefanutti D, Fitting JW. Sniff nasal inspiratory pressure. Reference values in Caucasian children. Am J Respir Crit Care Med 1999; 159: 107-111.

9 Zapletal A, Paul T, Samanek N. Die Bedeutung heutiger Methoden der Lungen-funktionsdiagnostik zur Feststellung einer Obstruktion der Atemwege bei Kindern und Jugendlichen [Significance of contemporary methods of lung function testing for the detection of airway obstruction in children and adolescents]. $Z$ Erkr Atmungsorgane 1977; 149: 343-371.

10 Robbins KR, Saxton AM., Southern LL. Estimation of nutrient requirements using broken-line regression analysis. J Anim Sci 2006; 84: Suppl., E155-E165.

11 Cole TJ, Bellizzi MC, Flegal KM, et al. Establishing a standard definition for child overweight and obesity worldwide: international survey. BMJ 2000; 320: 1240-1243.

12 Nicot F, Hart N, Forin V, et al. Respiratory muscle testing: a valuable tool for children with neuromuscular disorders. Am J Respir Crit Care Med 2006; 174: 67-74.

13 Tzelepis GE, Zakynthinos S, Vassilakopoulos T, et al. Inspiratory maneuver effects on peak expiratory flow. Role of lung elastic recoil and expiratory pressure. Am J Respir Crit Care Med 1997; 156: 1399-1404.

14 Pedersen OF. The Peak Flow Working Group: physiological determinants of peak expiratory flow. Eur Respir J 1997; 10: Suppl. 24, 11s-16s.

15 Omar T, Alawadhi H, Soubani AO, et al. Peak expiratory flow with or without a brief postinspiratory pause. Chest 2005; 128: 442-445.

\section{Low socioeconomic status is associated with worse lung function in the Danish cystic fibrosis population}

To the Editor:

Low socioeconomic status is associated with worse lung function and greater risk of death in people with cystic fibrosis (CF) in the UK and USA, but there are no population level studies from other countries [1-4]. A feature of previous analyses of inequalities in lung function in CF by socioeconomic status has been the identification of a lung function deficit in more disadvantaged children, which is evident as soon as spirometry can be routinely undertaken at $\sim 5-6$ years of age $[1,3]$. The finding of a fixed lung function deficit in the most deprived children in the early years of life has important policy implications, and suggests that deprivation has a detrimental effect on lung health in the early years of a child's life [5]. 